\title{
Quantum Measurement Strikes a Balance
}

\section{An experiment demonstrates that, in a quantum measurement, there is a three-way trade-off between different types of information content.}

\author{
By Rachel Berkowitz
}

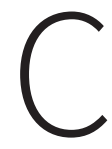

onventional theory says that making a quantum measurement changes the system irreversibly. But recent experiments indicate that a quantum state disturbed by a measurement can be recovered by a postmeasurement operation. Now, Hyang-Tag Lim, Seung-Woo Lee, and their colleagues at the Korea Institute of Science and Technology in Seoul demonstrate a scheme for testing different types of quantum measurements and determining how the total information is preserved [1]. They report that information about a quantum state is split into three distinct parts, in a quantifiable relationship that depends on the measurement strength.

The team's measurement scheme used a photonic qutrit, which is a three-level quantum system whose states are defined by a single photon's path modes. Optical components, including angled wave plates and beam splitters, both prepared the photon's states and implemented measurement operators. During each measurement, the researchers evaluated three

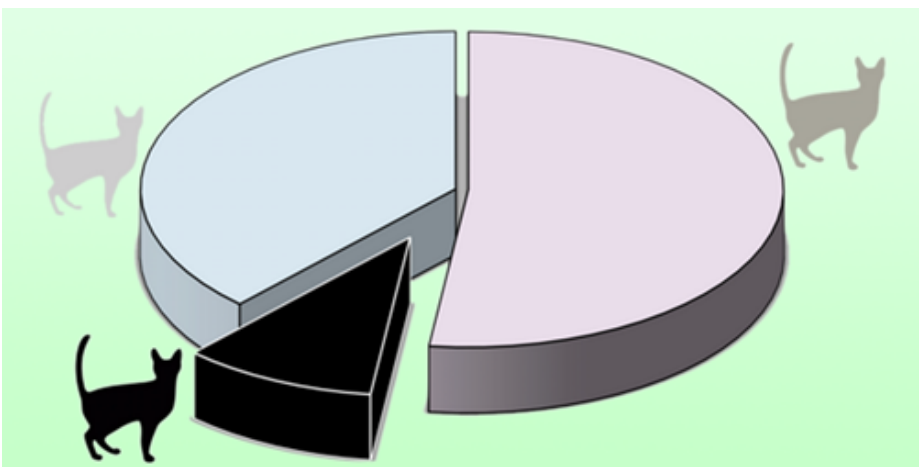

Credit: S. Hong et al. [1] types of information content: the amount of information gained by the observer about the qutrit state, the amount of undisturbed information actually transmitted from system to observer during the measurement, and the success probability of restoring the undisturbed state by applying a reverse operator. By varying the strength and types of measurement operators, the researchers observed that the amount of each type of information tended to vary, with one quantity's increase coming at the expense of another. They conclude that the information-disturbance relationship can be summarized as "the more information is obtained, the more the state is disturbed and the less recoverable it is."

The experiment adds to our understanding of the information conservation law in quantum mechanics and shows how quantum measurements could be optimized to preserve information. Such optimized measurements could help researchers build reliable quantum information technologies.

Correction (4 February 2022): The text was corrected, as it incorrectly stated that the work tested whether information is conserved rather than how the information is redistributed among different channels.

Rachel Berkowitz is a Corresponding Editor for Physics based in Vancouver, Canada.

\section{REFERENCES}

1. S. Hong et al., "Demonstration of complete information trade-off in quantum measurement," Phys. Rev. Lett. 128, 050401 (2022). 